\title{
Obstructive sleep apnea and dyslipidemia: pathophysiological mechanisms
}

\author{
Oladipupo Olafiranye • Ajibola Monsur Adedayo
}

Received: 1 October 2012 / Accepted: 3 October 2012 / Published online: 6 November 2012

(C) Springer-Verlag (outside the USA) 2012

\section{Dear Editor,}

We read with great interest the letter to the editor entitled "Obstructive sleep apnea and dyslipidemia: importance of the liver axis" [1] in response to our recent paper addressing dyslipidemia in obstructive sleep apnea (OSA) [2]. The authors emphasized the importance of the liver axis in the pathogenesis of OSA-induced dyslipidemia and referenced the theoretical possibility of the liver as a potential target for intervention. We are grateful for their interest in this topic which further underscores its importance.

We summarized the data on the liver pathways in the section on pathophysiological mechanisms and Fig. 1 of our paper [2]. However, the point is noted that OSA may be independently associated with insulin resistance and type- 2 diabetes as well. Although it has been proposed that induction of phospholipids and triglyceride production by hypoxia-inducible factor (HIF) in the liver is due to sterol regulatory element binding protein-1 (SREBP-1) independent of SREBP-2 which is responsible for cholesterol production via the 3hydroxy-3-methylglutaryl CoA reductase [3]. However, the mechanisms of HIF and SREBP-1 activation in the

O. Olafiranye $(\square)$

Heart and Vascular Institute,

University of Pittsburgh Medical Center,

Pittsburgh, PA, USA

e-mail: olafiranyeo@upmc.edu

O. Olafiranye $\cdot$ A. M. Adedayo

Brooklyn Health Disparities Center,

State University of New York Downstate Medical Center, Brooklyn,

New York, NY, USA liver by chronic intermittent hypoxia from OSA are not completely understood. While we also discussed the role of OSA-induced sympathetic activation in the development of dyslipidemia, we acknowledge that the role of hormone-sensitive lipase is central to peripheral lipolysis from adipose tissue which is responsible for most of the free fatty acid presented to the liver for lipoprotein generation [4].

Of note, we discussed multiple pathways including activation of the sympathetic autonomic nervous system that have been proposed for OSA-induced dyslipidemia [2], but most importantly, we emphasized that a direct cause and effect relationship is yet to be established. Therefore, there is a need for more studies to precisely define the relationship between OSA and dyslipidemia and determine potential targets for intervention.

\section{References}

1. Mirrakhimov AE, Mirrakhimov EM (2012) Obstructive sleep apnea and dyslipidemia: importance of the liver axis. Sleep Breath. doi: 10.1007/s11325-012-0772-5.

2. Adedayo AM, Olafiranye O, Smith D, Hill A, Zizi F, Brown C, Jean-Louis G (2012) Obstructive sleep apnea and dyslipidemia: evidence and underlying mechanism. Sleep Breath. doi:10.1007/ s11325-012-0760-9O, PMID:22903801

3. Li J, Thorne LN, Punjabi NM, Sun CK, Schwartz AR, Smith PL, Marino RL, Rodriguez A, Hubbard WC, O'Donnell CP, Polotsky VY (2005) Intermittent hypoxia induces hyperlipidemia in lean mice. Circ Res 97(7):698-706

4. Kraemer FB, Shen WJ (2002) Hormone-sensitive lipase: control of intracellular tri-(di-)acylglycerol and cholesteryl ester hydrolysis. J Lipid Res 43(10):1585-1594 